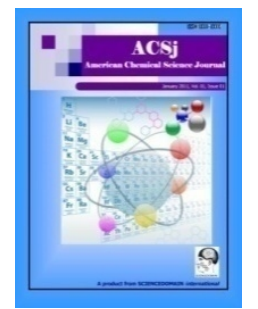

American Chemical Science Journal

2(2): 38-44, 2012

SCIENCEDOMAIN international

www.sciencedomain.org

\title{
Production and Visualization of Vivid Streakline with the Self-Assembly of Surfactant Molecules
}

\author{
Shunsuke Hashimoto $^{1^{\star}}$ and Akitoshi Nishimura ${ }^{1}$ \\ ${ }^{1}$ Division of Chemical Engineering, Department of Materials Engineering Science, \\ Graduate School of Engineering Science, Osaka University, 1-3 Machikaneyama, Toyonaka, \\ Osaka 560-8531, Japan. \\ Authors' contributions \\ This work was carried out in collaboration between all authors. SH designed the study, \\ performed and managed experiments, wrote the protocol, and wrote all the part of the \\ manuscript. AN also performed and supported experiments. All authors read and approved \\ the final manuscript.
}

Research Article

Received $8^{\text {th }}$ May 2012

Accepted $11^{\text {th }}$ June 2012

Online Ready $13^{\text {th }}$ June 2012

\section{ABSTRACT}

Aims: Streakline and its visualization and production techniques are one of important indices for fluid dynamics, civil engineering, material engineering, and so on. In chaotic or turbulent flow field where global mixing occurs, fluid trajectory exponentially expands in particular directions. Consequently, it is quite difficult to visualize streak as a "line" because experimental streak behaves as a "sheet" that has rounded surface. In the present study, to product and visualize a vivid and distinct streakline, a non-ionic surfactant was utilized.

Study Design: Experimental study containing simulation.

Place and Duration of Study: Division of Chemical Engineering, Department of Materials Engineering Science, Graduate School of Engineering Science, Osaka University, between December 2010 and October 2011.

Methodology: The novel production and visualization technique for vivid streakline in three-dimensional (3D) flow field was developed. The innovative characteristic of visualization technique is that the expansion of streak can be suppressed by the surface tension with the self-assembly of surfactant molecules in the tracer fluid. The impelleragitated tank was adopted as an unsteady 3D flow system. A non-ionic surfactant, "Emulgen 103 (Kao Co., Ltd., polyoxyethylenelaurylether) was adopted, whose 
concentration was optimized at about 9 mass $\%$ in advance.

Results: The obtained streakline could keep the linear form for a long time not to expand, and there was little leakage of colored component due to the effect of molecular diffusion. The streakline could circulate freely in the tank; nevertheless its linear structure was maintained for a long time even if the streakline collided with the tank wall and impeller blades. The periodical consistency of streakline structure could be confirmed, which was one of necessary and sufficient conditions for the movement of streakline with flow field.

Conclusion: This study clearly demonstrates the production method of vivid streakline with the help of surfactant. While this technique has a little room for additional refinement, the present technique may be used and developed for future science and practical applications.

Keywords: Chaotic advection; Non-linear Dynamics; Streakline; Surfactant; Self-assembly; Visualization.

\section{INTRODUCTION}

Fluid flow is described in general by a vector field in three (for steady flows) or four (for unsteady flows with respect to time) dimensions. Pathlines, streamlines and streaklines are three important field lines of different vector-field descriptions of the flow (Batchelor, 1999; Hama, 1962). Streamlines are a mass of curves that are instantaneously tangent to the velocity vector of the flow. This means that if a point is picked, and then the flow moves in a certain direction at that point. Moving a small distance along this direction and then finding out where the flow now points would draw out a streamline. Pathlines are the trajectory that a fluid particle would make as it moves around with the flow. Streaklines are the locus of points of all the fluid particles that have passed continuously though a particular spatial point in the past. This can be found experimentally by releasing dye into the fluid in a time period at a fixed point and then at a later time finding out where the dye was. By definition, streamlines defined at a single instant in a flow do not intersect with one another. This is so because a fluid particle cannot have two different velocities at the same point. Similarly, streaklines cannot intersect themselves or other streaklines, because two particles cannot be present at the same location at the same instance of time. However, pathlines are allowed to intersect themselves or other pathlines (except the starting and end points of the different pathlines, which need to be distinct). In simple terms, streamlines and streaklines are like a snapshot of the flow field whereas pathlines are time-history of a flow.

In steady flow (which flows that remain the same for all time, for example, tubular flow), the streamlines, pathlines, and streaklines coincide with one another. This is useful, because it is usually very difficult to visualize streamlines in an experiment. However, in the case with the steady flow, streaklines can be utilized to describe the streamline pattern. This is why streaklines are highly available as an index on fluid analysis.

On the other hand, in unsteady flow systems such as three-dimensional stirred tanks, the flow changes with respect to time and each particle will go in different directions. Hence, it is difficult to predict and visualize the streakline structure because the configuration of streak is significantly different from those of streamline and pathline (Hama, 1962). The trajectory of fluid particles drastically metamorphoses depending on their initial positions. Additionally, in 
convective-flow fields containing both chaotic and turbulent-flow regions where global mixing occurs, the fluid trajectory tends to expand exponentially in a particular direction, and it is therefore difficult experimentally to visualize a streak as a "line" because the streak behaves as a "sheet" that has rounded surface (Inoue et al., 2009).

Streaklines are very important index on the region of fluid dynamics. Mixing is one of important unit operations on the chemistry and/or chemical engineering, which can often dominate the efficiency of chemical reaction in a reactor tank. In this case, it has been revealed that streaklines play an important role as a forceful index that controls the performance of convective mixing (Inoue and Hashimoto, 2010; Hashimoto et al., 2011). Furthermore, streakline is significant for various research regions: its visualization is essential for fluid dynamics as well as civil and mechanical engineering to predict stream and its production is useful for material synthesis and engineering to prepare fine linear and/or curved plastic resins. There are almost no methods for streakline production and visualization except for a complicated technique using neutron scattering (Cimbala and Sathianathan, 1988). Thus, the technique of visualization and production for vivid streakline with simple methods is expected.

This study provides novel technique for the visualization of vivid and distinct streaklines with a new method of streakline production. With this method, the vivid streakline can be produced and visualized for a long time in spite of the unsteady flow system in stirred tank. The obtained streakline remains stable at least for 50 periods $(50 \mathrm{~T})$ of impeller-blade rotation. In this method, the self-assembly and distribution of surfactant molecules are utilized with hydrophobic-hydrophilic interaction. In general, the addition of shear energy to the fluid including surfactant results in the self-organization of surfactant molecules, which produces the effect of drag reduction in steady flow systems (Yunying and Jacques, 2002).

\section{MATERIALS AND METHODS}

Glycerol aqueous solution (80 mass\%, from deionized water) was prepared as a bulk fluid whose viscosity and density were $0.046 \mathrm{~Pa} \cdot \mathrm{s}$ and $1197.7 \mathrm{~kg} / \mathrm{m}^{3}$ at $298 \mathrm{~K}$, respectively. Glycerol aqueous solution was generally known as a Newtonian fluid. As a tracer fluid, pure glycerol was used with the desired amount of methylene blue (3, 7-bis (Dimethylamino)phenothiazin-5-ium chloride) dye. Then, a non-ionic surfactant, "Emulgen 103 (Kao Co., Ltd., polyoxyethylenelaurylether; Information of Emulgen is available at http://chemical.kao.com/jp/)", which is well known as one of cosmetic emulsifiers and its surface tension is $1.26 \mathrm{~N} / \mathrm{m}$. This surfactant was suspended and dispersed in the tracer fluid at ca. 9 mass\% without counter ions. The viscosity and density of tracer fluid were slightly higher than those of bulk one, while its difference was at most less than $3 \%$. In advance, the rheological properties of the fluid with surfactant have been investigated by use of stresscontrol-type rheometer (Anton Paar, MCR302). As a result, the present tracer fluid containing glycerol and Emulgen 103 exhibits the Newtonian behavior under the conditions of shear rate from 5 to $3000 \mathrm{~s}^{-1}$. Thus, the present amount of surfactant did not affect the Newtonian property of tracer fluids.

The production and visualization experiments of streaklines were performed by use of impeller agitating flow in a stirred tank as a representative for unsteady and complicated flow, where both rotating and swirling flows generate. The two-bladed paddle impeller was adopted, which was well known as one of quite simple impellers. One of impeller blades was fabricated so that the tracer fluid could be injected into the tank from the tip of the impeller blade. The impeller was installed on the center of the tank. In this case, the fluid conditions 
are generally defined as agitating Reynolds number: $R e=\rho n_{\mathrm{r}} d^{2} /$. The nomenclatures, $\rho$ and stand for density and viscosity of bulk fluids, respectively, and $n_{\mathrm{r}}$ and $d$ stand for rotation speed and diameter of impeller blade. Definitely, the region of $R e<10$ is considered as a gentle flow (laminar-flow conditions), and that of $10<R e<1500$ is transition to turbulent flow where both laminar and turbulent flows exist at the same time. In this study, the production and visualization experiments of streaklines were performed in the range of 10 $<R e<150$. The tracer fluid was injected into bulk fluid from the tip of an impeller blade at the volume flow rate of $3.33 \times 10^{-8} \mathrm{~m}^{3} / \mathrm{s}$ by use of syringe pump, which was small enough not to affect surrounding fluid conditions. To stabilize streakline at $R e>150$, utilizing another surfactants that have larger surface tension than Emulgen, for example, some cationic or anionic surfactant may be required. However, further investigation will be required.

\section{RESULTS AND DISCUSSION}

Fig. 1 shows the typical images of visualized streaks. As can be seen in Fig. 1(a), the streakline expands exponentially to vertical directions and then it creates sheeted structure and becomes vague fast without surfactant. On the other hand, as shown in Fig. 1(b), we have succeeded in producing and visualizing the vivid and distinct streakline under unsteady flow conditions. Surprisingly, this streakline can circulate freely in the tank; nevertheless its linear structure is maintained for a long time even if the streakline collide with the tank wall and impeller blades. Although the streakline becomes vague gradually after $t=100 T$, Fig. 2 indicates that the periodical consistency of streakline structure can be confirmed, which is one of necessary and sufficient conditions for the movement of streakline with flow field (Batchelor, 1999). After further time passes, the bulk fluids in the tank are blue-colored by degrees, while its coloring rate is extremely small. This suggests that the suppressive effect of surfactant on molecular diffusion becomes sustained for a long time. It has been confirmed that the present technique for the production and visualization of streakline are workable under the wide conditions of at least $R e<150$.
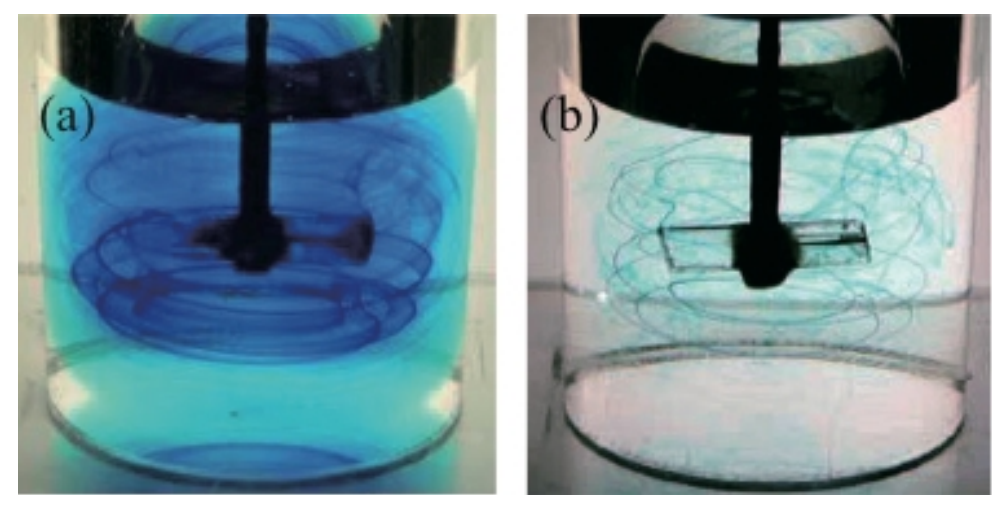

Fig. 1. Experimental images of three-dimensional streak generated from the tip of paddle impeller blade in stirred tank at $R e=60, t=30 T$; (a) streak sheet (without surfactant), (b) streakline (with surfactant). $T$ stands for the rotational period of impeller blade 


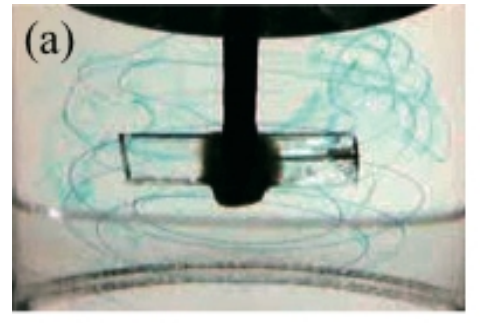

$20 T$

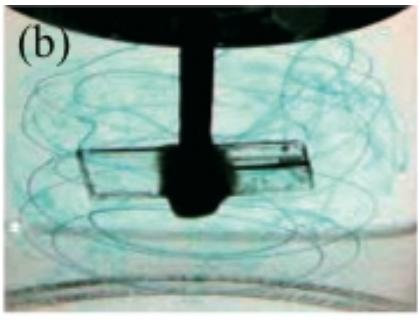

$30 T$

Fig. 2. Periodical variation of three-dimensional experimental streakline generated from the tip of an impeller blade in stirred tank $(R e=60)$; (a) $t=20 T$, (b) $t=30 T$

One of possible reasons for successfully production of vivid streakline here is the suppressive effect on the stretch of tracer and molecular diffusion from the column of tracer fluid, which is due to the self-assembly of non-ionic surfactant molecules at the surface of the column of tracer fluid (Srinivas et al., 2006). In general, surfactant molecules are selforganized in some porous materials (Müter et al., 2010). In this study, to make surfactant molecules oriented in the same direction, the moisture content in bulk fluids is adjusted at 20 mass \%, while the tracer fluid contains no water. As can be seen in Fig. 3, this enables surfactant molecules to line up around the column of tracer fluid by hydrophobic-hydrophilic interaction. Surprisingly in this study, surfactant molecules remain the alignment state for a long time even if they are in unsteady flow fields without surrounding matrix structures. It is likely that streakline cannot follow the flow field because of the stiffness by the excess thickness of surfactant. Hence, it is important to optimize the concentration of surfactant. The present experimental streakline is qualitatively coincident with the simulated one (Inoue et al., 2009). Under the present conditions, the streakline may successfully move on the flow field. Additionally, to linearize the streakline efficiently, the outflow rate of tracer fluid also has to be adjusted. Theoretically, the streakline generated from a nozzle is considered as a liquid-column jet, and then various mechanical unstable phenomena such as Rayleigh instability (Rayleigh, 1878; Weber, 1931) come into existence. Hence, it is essential for successful production and visualization of streakline to set up the concentration of surfactant (that is, surface tension) as well as the relative ratio of viscosity and/or density between bulk and tracer fluids, their relative velocity, the diameter and shape of nozzle in advance. In fact, in preliminary experiments, the streakline becomes sheet-like structure at low concentration (< 7 mass \%) of surfactant, while liquid drops of surfactant are observed at high concentration (> 11 mass \%) of surfactant. Thus, streakline can be obtained under the narrow conditions, where the period of preservation of linear form is ca. $60 T$ (surfactant concentration: 8,10 , and 11 mass \%) and $100 T$ (9 mass\%, optimal). The high injection speed of tracer fluid also results in the sheet-like expansion of streakline. Needless to say, it has been confirmed that the streakline can be produced in the steady flow such as tubular flow by use of the present technique. Finally, one of measures to stabilize streakline at Re> 150 is utilizing another surfactants that have larger surface tension than emulgen, for example, some cationic or anionic surfactant. However, further investigation will be required. 
(a) without surfactant

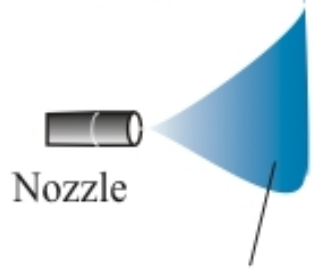

(b) with surfactant

Streak sheet

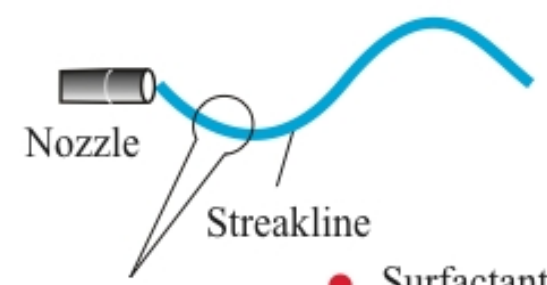

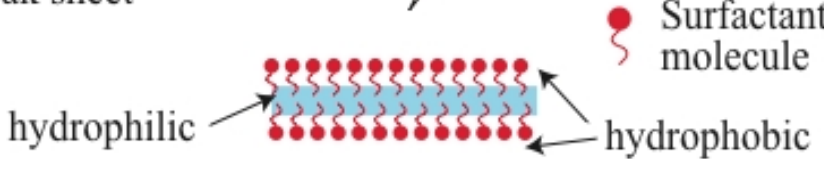

Fig. 3. Conceptual image of streak; (a) streak sheet without surfactant, (b) streakline surrounded by surfactant molecules.

\section{CONCLUSION}

In the present study, the novel production and visualization technique for vivid streakline in three-dimensional flow field was developed. The innovative characteristic of visualization technique is that the expansion of streak can be suppressed by means of surface tension of self-organized surfactant molecules in the tracer fluid. The obtained streakline can keep the form of a line for a long time without sheeted expansion, and there is little leakage of colored component due to the effect of molecular diffusion.

While this technique has a little room for additional refinement, this study clearly demonstrates the production method of vivid streakline with the help of surfactant, which may be used and developed for future science and practical applications.

\section{ACKNOWLEDGEMENTS}

This work was partially supported by Grant-in Aid for Scientific Research, A (20246115) and Challenging Exploratory Research (22656176).

\section{COMPETING INTERESTS}

Authors have declared that no competing interests exist.

\section{REFERENCES}

Batchelor, G.K. (1999). An introduction to fluid dynamics 1999, Cambridge Univ. Press, New York. N.Y.

Cimbala, J.M., Sathianathan, D. (1988). Streakline flow visualization with neutron radiography, Exp. Fluids, 6, 547-552.

Hama, F.R. (1962). Streaklines in a perturbed shear flow. Phys. Fluids, 5, 644-650. 
Hashimoto, S., Natami, K., Inoue, Y. (2011). Mechanism of mixing enhancement with baffles in impeller-agitated tank, part I: a case study based on cross-sections of streak sheet. Chem. Eng. Sci., 66, 4690-4701.

Inoue, Y., Okada, B., Hashimoto, S. (2009). Simplified model of 3D velocity field in stirred tank. Kagaku KogakuRonbunshu, 35, 201-210.

Inoue, Y., Hashimoto, S. (2010). Analysis of mechanism of laminar fluid mixing in 3-D mixing tank based on streakine lobes. Kagaku KogakuRonbunshu, 36, 355-365.

Müter, D., Shin, T., Demé, B., Fratzl, P., Paris, O., Findenegg, G.H. (2010). Surfactant selfassembly in cylindrical silica nanopores. J. Phys. Chem. Lett., 1, 1442-1446.

Rayleigh, L. (1878). On the instability of jets. Proc. London Math. Soc., 10, 4-13.

Srinivas, G., Nielsen, S.O., Moore, P.B., Klein, M.L. (2006). Molecular dynamics simulations of surfactant self-organization at a solid-liquid interface. J. Am. Chem. Soc., 128, 848-853.

Weber, C. (1931). Disintegration of liquid Jets, Z. Angew. Math. Mech., 1, 136-159.

Yunying, Q, Jacques, L.Z. (2002). Chemical and rheological characterization of dragreducing cationic surfactant systems, Ind. Eng. Chem. Res., 41, 6326-6336.

(c) 2012 Hashimoto \& Nishimura; This is an Open Access article distributed under the terms of the Creative Commons Attribution License (http://creativecommons.org/licenses/by/3.0), which permits unrestricted use, distribution, and reproduction in any medium, provided the original work is properly cited. 